\title{
Melanoma antigen A12 regulates cell cycle via tumor suppressor p21 expression
}

\author{
Teruki Yanagi ${ }^{1,2}$, Ko Nagai ${ }^{1}$, Hiroshi Shimizu ${ }^{2}$ and Shu-Ichi Matsuzawa ${ }^{1,3}$ \\ ${ }^{1}$ Sanford Burnham Prebys Medical Discovery Institute, La Jolla, California, USA \\ ${ }^{2}$ Department of Dermatology, Hokkaido University Graduate School of Medicine, Sapporo, Japan \\ ${ }^{3}$ Department of Neurology, Kyoto University Graduate School of Medicine, Kyoto, Japan \\ Correspondence to: Shu-lchi Matsuzawa, email: smatsuza@kuhp.kyoto-u.ac.jp \\ Keywords: MAGEA 12, cell cycle, p21, cyclin dependent kinase, ubiquitination \\ Received: May 09, $2017 \quad$ Accepted: June 08, $2017 \quad$ Published: July 22, 2017 \\ Copyright: Yanagi et al. This is an open-access article distributed under the terms of the Creative Commons Attribution License \\ 3.0 (CC BY 3.0), which permits unrestricted use, distribution, and reproduction in any medium, provided the original author and \\ source are credited.
}

\section{ABSTRACT}

Melanoma-associated antigen family A (MAGE-A) is a family of cancer/testis antigens that are expressed in malignant tumors but not in normal tissues other than the testes. MAGE-A12 is a MAGE-A family gene whose tumorigenic function in cancer cells remains unclear. Searches of the Oncomine and NextBio databases revealed that malignant tumors show up-regulation of MAGE-A12 mRNA relative to corresponding normal tissue. In PPC1 primary prostatic carcinoma cells and in HCT116 colorectal cancer cells (wild type and p53-depleted), MAGE-A12 gene knockdown using siRNA or shRNA diminishes cancer cell proliferation as assessed by cellular ATP levels, cell counting, and clonogenic assays. FACS analyses of annexin V-PI staining and DNA content show that MAGE-A12 knockdown causes G2/M arrest and apoptosis. In tumor xenografts of HCT116 cells, conditional knockdown of MAGE-A12 suppresses tumor growth. The depletion of MAGE-A12 leads to the accumulation of tumor suppressor p21 in PPC1, HCT116, and p53-depleted HCT116 cells. Conversely, CDKN1A knockdown partially rescues the viability of PPC1 cells transfected with siRNA targeting MAGE-A12, while p21 overexpression leads to proliferation arrest in PPC-1 cells. Furthermore, exogenous MAGE-A12 expression promotes the ubiquitination of p21. Our findings reveal that MAGE-A12 plays crucial roles in p21 stability and tumor growth, suggesting that MAGE-A12 could provide a novel target for cancer treatment.

\section{INTRODUCTION}

The melanoma-associated antigen (MAGE) protein family is a large, highly conserved group of proteins that share a MAGE homology domain [1]. The human MAGE family proteins can be divided into two groups based on expression pattern. Type I MAGE proteins are cancer/ testis antigens and include the MAGE-A, $-\mathrm{B}$, and -C subfamilies, which are clustered on the $\mathrm{X}$-chromosome. Type II MAGE proteins are expressed throughout tissues and are not limited to the $\mathrm{X}$ chromosome [1]. Members of melanoma-associated antigen family A (MAGE-A) are expressed in a wide variety of malignant tumors but not in normal adult tissues other than the testes [2]. Higher
MAGE-A expression correlates with a poor outcome for patients with certain cancers, such cancers of the breast, prostate, and lungs [3-7]. The expression of MAGE-A family members is increased in cancer stem-like cell populations [8]. Recently, some MAGE-A proteins have been reported to regulate the ubiquitination of the $\mathrm{p} 53$, thereby regulating cancer cell proliferation [9-11]. However, most of the functions of MAGE-A proteins have not been investigated in detail. MAGE-A12, a MAGE-A family gene, has similar amino acid sequences to other MAGE-A family proteins, especially MAGE-A2, -A3, and A-6 [2]. MAGE-A12 is expressed in malignant tumors, but not in non-neoplastic normal tissues [12, 13]; however, its tumorigenic function remains unclear. 
p21 (also known as Cip1, Waf1, cyclindependent kinase inhibitor 1A) is a tumor suppressor that regulates cell proliferation [14]. p21 binds to and inhibits the kinase activity of Cdk2 and Cdk1, leading to cell cycle arrest. Furthermore, p21 is associated with not only G2 arrest but also senescence [15]. In contrast to the conventional tumor suppressor p53, loss of p21 expression commonly occurs not through genetic mutation, but through transcriptional or posttranscriptional regulation. Much of the control of p21 expression is at the transcriptional level, especially p53-dependent regulation. p21 expression is also controlled via post-transcriptional ubiquitin-dependent or independent proteolysis; however, the details of the mechanisms have not been elucidated.

We here in report here investigations on the role of MAGE-A12 in tumorigenesis that provide evidence that MAGE-A12 plays a crucial role in the proliferation of some types of cancer cells. $M A G E$ - $A 12$-depleted cancer cells show $\mathrm{G} 2 / \mathrm{M}$ phase arrest, followed by apoptosis. $M A G E-A 12$ knockdown leads to the accumulation of tumor suppressor p21 without mRNA up-regulation. Knockdown of $C D K N 1 A$ rescues the cell viability of cancer cells transfected with siRNA targeting MAGE-A12. Moreover, CMV promoter driving p21 overexpression leads to proliferation arrest in PPC1 cells. In tumor xenografts, the conditional knockdown of $M A G E-A 12$ suppresses tumor growth. Taken together, these findings reveal an unexpected role for MAGE-A12 in cancer cell proliferation, suggesting that these molecules may provide novel targets for the future discovery of oncology therapies.

\section{RESULTS}

\section{MAGE-A12 is overexpressed in malignant tumors and associated with poor patient- prognosis}

To assess the role of MAGE-A12 in normal tissues and cancers, we examined a public database to evaluate the levels of $M A G E-A 12$ expression. The Oncomine database (https://www.oncomine.org/) showed MAGE-A12 to be expressed only in the testes and not in any other organs (Supplementary Figure 1), which is consistent with previous studies showing that MAGE-A family genes are expressed in only cancer or the testes. The NextBio database (https://www.nextbio.com/), a TCGA database containing information on $M A G E-A 12$ RNA expression levels, showed $M A G E-A 12$ to be an up-regulated gene in cancers, relative to corresponding normal tissue (Supplementary Figure 2A). The prognostic value of $M A G E-A 12$ was assessed using the Kaplan-Meier Plotter (http://kmplot.com/analysis/), an online tool to correlate survival with gene expression, based upon microarray data from 1,405 patients with lung cancer. High $M A G E-A 12$ mRNA levels were significantly correlated with lower overall survival in lung cancer (Supplementary Figure 2B). Similar results were observed in the patients with gastric cancer (Supplementary Figure 2C). This data from public databases indicates that MAGE-A12 is over-expressed in malignant tumors and is associated with poor patient-prognosis. Next, we assessed MAGE-A12 mRNA expression levels in various cell lines. Compared to normal cell lines (IMR-90 and 267B1 cells), two cancer cell lines (HCT116 and PPC1) showed higher MAGE-A12 expression levels (Supplementary Figure 3).

\section{Knockdown of MAGE-A12 regulates tumor cell growth and proliferation}

To investigate the effect of MAGE-A12 knockdown in cancer cells, we performed siRNA experiments using two siRNAs that target MAGE-A12. In human PPC1 primary prostatic cancer cells, quantitative RT-PCR and immunoblotting confirmed reduced levels of mRNA and protein by these siRNAs (Figure 1A and 1B). First, we analyzed cell viability changes in the presence of MAGE-A12 knockdown. Prostate cancer PPC1 cells were treated with negative control siRNAs or siRNAs targeting MAGE-A12, and cell viability was assessed 72 hours later. Cultures of MAGE-A12 knockdown PPC1 cells showed reduced relative numbers of viable cells compared to control cell cultures transfected with negative control siRNA, measured 3 days after transfection using ATP levels (Figure 1C). Similar results were obtained by direct cell counting methods, showing a reduction in the numbers of viable cells in cultures of PPC1 cells within 3 days of MAGE-A12 siRNA treatment (Figure 1D).

Next, to support the results of the siRNA experiments, we performed shRNA experiments in which the target sequences of shRNAs were different from those of the siRNAs. We used tetracycline-inducible shRNA vectors targeting MAGE-A12 to assess the impact of MAGE-A12 deficiency on the growth and survival of tumor cells. In PPC1 cells that were stably infected with a lentivirus expressing two different shRNAs targeting MAGE-A12, culturing with the tetracycline analog doxycycline resulted in reductions in MAGE-A12 mRNA levels (Figure 1E). Doxycycline-inducible reductions in MAGE-A12 protein were also observed (Figure 1F). In contrast, MAGE-A12targeting shRNAs did not substantially reduce levels of mRNAs encoding MAGE-A2, -A3, or -A6 (Supplementary Figure 4). Compared to control cells (Tet-OFF), the growth rates of doxycycline-stimulated MAGE-A12 knockdown PPC-1 cells (Tet-ON) were significantly diminished at 5 days after seeding (Figure 1G). In clonogenic assays, the number of tumor cell colonies was significantly lower with MAGE-A12 knockdown (Tet-ON) than for control cells (Tet-OFF) (Figure 1H). Similar results were obtained using other tumor cell lines, such as human HCT116 colorectal cancer cells and isogenic p53-deficient cells (HCT116 
A
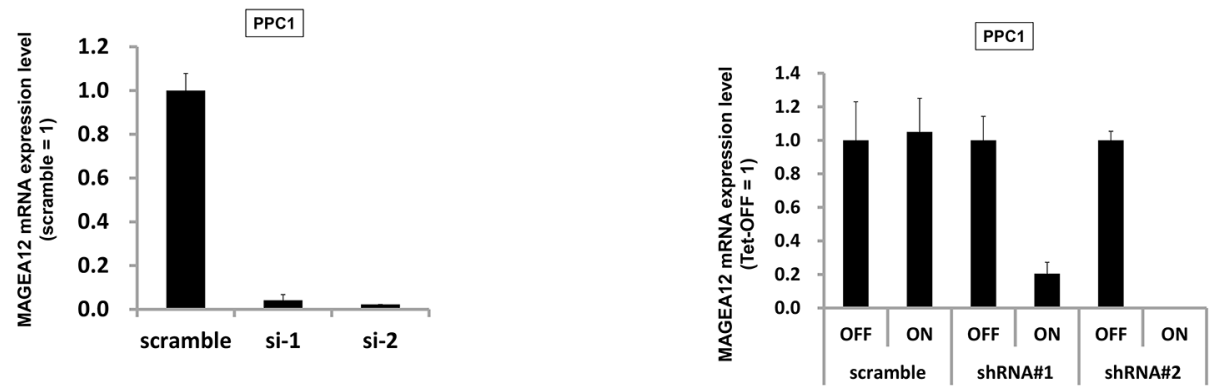

B

PPC1
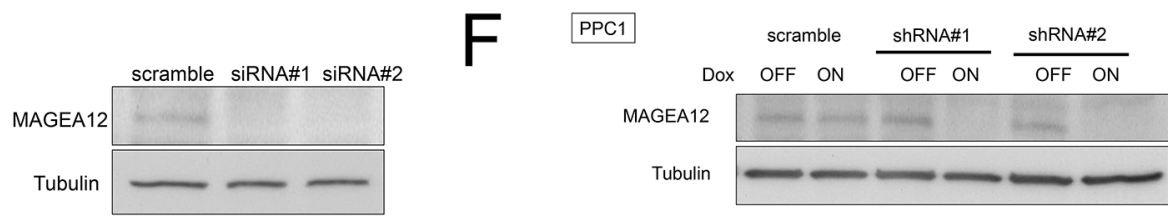

C
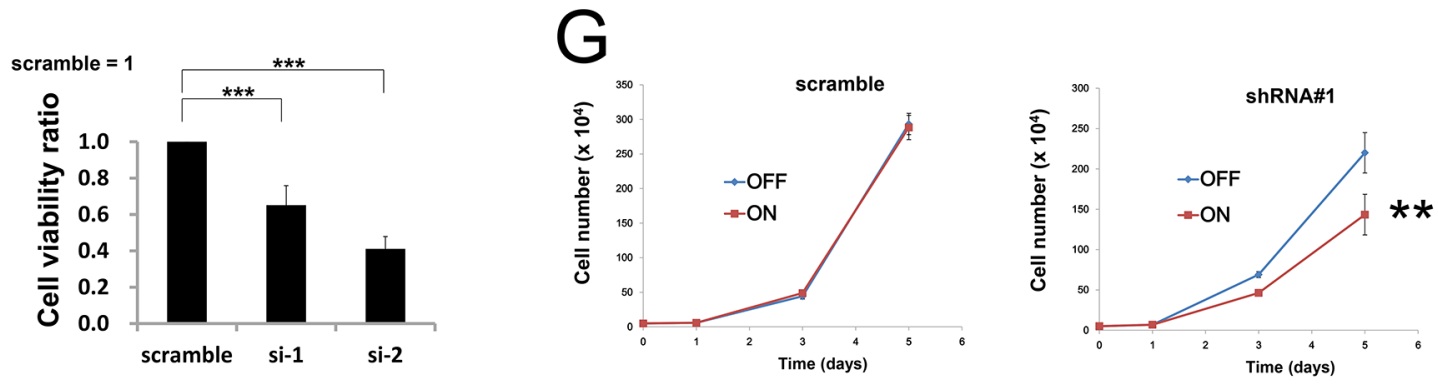

$\mathrm{D}$
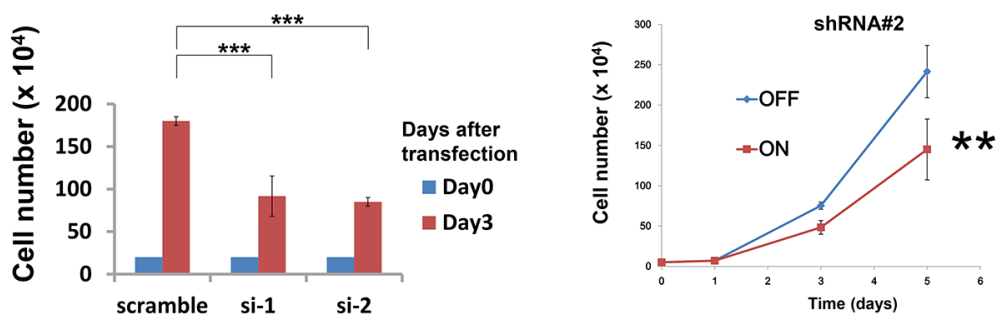

$\mathrm{H}$

Figure 1: Knockdown of MAGE-A12 mitigates the growth of cancer cells. (A) PPC1 cells transfected with scrambled RNA or two different siRNAs targeting MAGE-A12 (siRNAs\#1, \#2). After 48 hours, the relative levels of MAGE-A12 mRNA were measured by qRT-PCR analysis. (B) Cell lysates 48 hours after siRNA transfection were prepared and were normalized for total protein content, and aliquots were analyzed by immunoblotting using mouse anti-MAGE-A12 (top) or anti-beta-actin (bottom) antibodies. (C) PPC1 cells transfected with control RNA or various siRNAs targeting MAGE-A12. At 72 hours, cellular ATP levels were measured as a surrogate indicator of relative number of viable cells, with data expressed as the ratio of values for cells transfected with MAGE-A12 to values for the control siRNAs (mean $\pm \mathrm{SD} ; \mathrm{n}=3$ ). $* * * \mathrm{p}<0.001$ by t-test. (D) To measure cell growth, $2.0 \times 10^{5}$ cells transfected with the indicated siRNAs were seeded onto 60 -mm-diameter plates. At 72 hours, the numbers of cells were counted. *** p $<0.001$ by t-test. (E) PPC1 cells stably containing inducible shRNAs targeting different sites on MAGE-A12 mRNA (shRNA\#1, \#2) were cultured for 48 hours with doxycycline (Dox: $100 \mathrm{ng} / \mathrm{ml}$ ). Levels of MAGE-A12 mRNA were measured by qRT-PCR, with normalization relative to GADPH (mean $\pm \mathrm{SD} ; \mathrm{n}=2$ ). (F) Protein lysates were generated from PPC1 cells (shRNA\#1, \#2, and scramble control) that had been cultured for 48 hours with or without $100 \mathrm{ng} / \mathrm{ml}$ doxycycline, normalized for total protein concentration, and analyzed by SDS-PAGE/immunoblotting using antibodies for MAGE-A12 (top) and beta-actin (bottom). (G) PPC1 cells (5.0 x 104) stably containing inducible shRNAs were cultured in 60 -mm-diameter plates for 24 hours and then stimulated with (ON) or without (OFF) $100 \mathrm{ng} / \mathrm{ml}$ doxycycline. The cells were counted at 1 , 3 , and 5 days after seeding (mean $\pm \mathrm{SD} ; \mathrm{n}=3$ ). $* * \mathrm{p}<0.01$ by t-test. $(\mathbf{H})$ PPC1 cells stably containing inducible shRNAs were seeded at 300 cells per well in 60-mm dishes. At 24 hours, doxycycline (ON: $100 \mathrm{ng} / \mathrm{ml}$, OFF: $0 \mathrm{ng} / \mathrm{ml}$ ) was added. Colonies consisting of $>50$ cells were enumerated on day 10 . All data represent mean $\pm \mathrm{SD}(\mathrm{n}=3)$. ${ }^{* * *} \mathrm{p}<0.001$ by t-test. 
p53-) (Supplementary Figures 5, 6). From these results, we conclude that MAGE-A12 regulates the proliferation and survival of cancer cells. To extend these studies into an in vivo context, we used HCT116 cells containing inducible MAGE-A12 shRNA (\#2, doxycycline-induced knocked-down mRNA levels in shRNA\#2 were as low as those in shRNA\#1) in a tumor xenograft model. Immunocompromised null/null ( $\mathrm{nu} / \mathrm{nu})$ mice were injected subcutaneously with HCT116 cells, and tumors were allowed to grow for 11 days before doxycycline was added to the drinking water for 17 days to induce the shRNA vector, which resulted in reduced MAGE-A12 mRNA expression in tumors (Figure 2A). Inducing MAGE-A12 shRNA expression remarkably suppressed tumor growth in vivo (Figure 2B-2D).

\section{Knockdown of MAGE-A12 results in G2/M arrest and apoptosis}

To examine the mechanisms of cell growth suppression by MAGE-A12 knockdown, a fluorescent annexin $\mathrm{V} /$ propidium iodide (PI) double-staining assay was performed, which showed increases in apoptosis over time in cultures of MAGE-A12 RNAi-treated PPC1 cells (Figure 3A). Cell cycle analyses were also performed using FACS-based DNA content analysis of PPC1 cells, which allowed the percentages of $2 \mathrm{~N}, 4 \mathrm{~N}$, polyploid (DNA content $>4 \mathrm{~N}$ ), and hypoploid cells to be quantified (Figure 3B). In cultures of MAGE-A12 knockdown cells, the proportion of $\mathrm{G} 2 / \mathrm{M}$ phase cells (4N DNA content) and the number of hypoploid (apoptotic) cells were increased at 72 hours. SDS-PAGE/immunoblot analyses using MAGE-A12 knockdown PPC1 cell lysate showed that cdc25C and phospho-Cdk1 (Y15) levels were downregulated, which is consistent with FACS-based DNA content analysis (Figure 3C).

\section{MAGE-A12 knockdown leads to p21 accumulation}

To explore the mechanism by which knockdown of MAGE-A12 induces G2/M cell cycle arrest and apoptosis, we examined the impact of MAGE-A12 knockdown on the expression of various proteins that have been implicated in apoptosis and the cell cycle. siRNA or shRNA-mediated MAGE-A12 knockdown up-regulated expression levels of tumor suppressor p21 in PPC1, HCT116 cells, and HCT116 p53- cells (Figure 4A), while p21 mRNA levels were not notably up-regulated (Figure 4B). p21 accumulation was also observed in the shRNA-mediated MAGE-A12 knockdown cells (Supplementary Figure 7). In the HCT116 cells, MAGE-A12 knockdown also led to the upregulation of $\mathrm{p} 53$, which is consistent with the function of other MAGE-A family proteins [9]. These results suggest that the elevated levels of $\mathrm{p} 21$ protein expression by MAGE-A12 knockdown have both a p53-dependent pathway and a p53-independent pathway. Next, we assessed the effect of MAGE-A12 on p21's interaction with Cdks, which p21 is known to bind and suppress. The p21 protein that accumulated in MAGE-A12 knockdown cells was found to interact with Cdk1 and
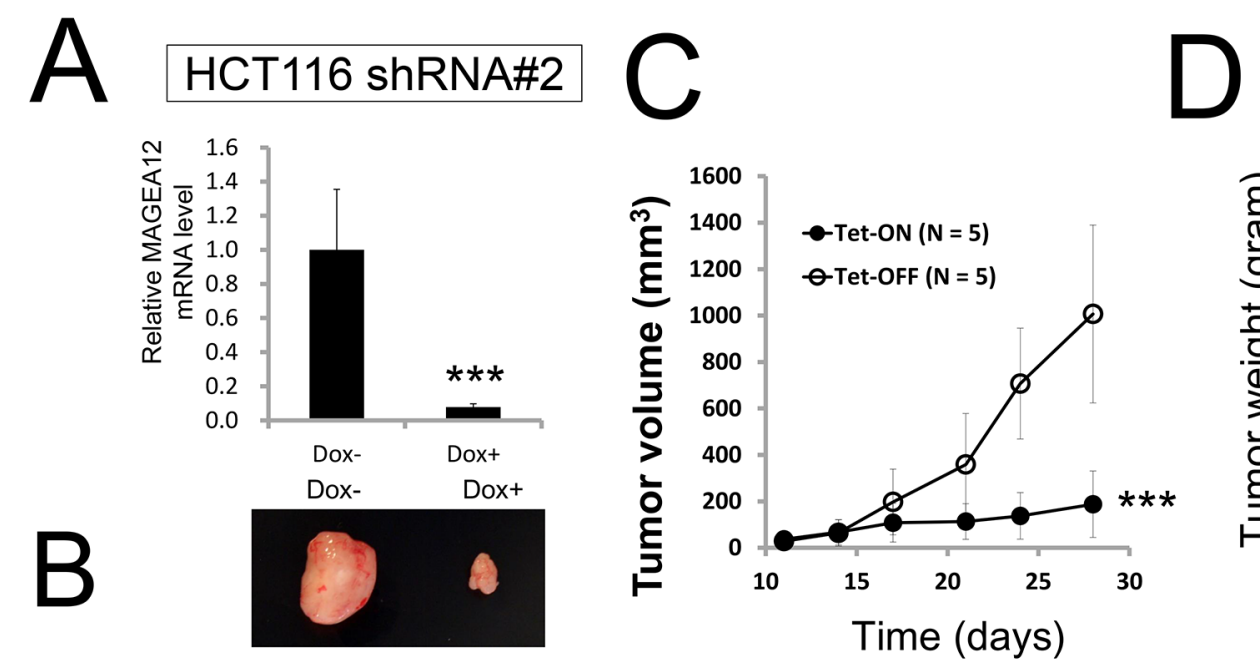

Figure 2: Knockdown of MAGE-A12 diminishes cancer cell growth in vivo. (A-D) In vivo tumor xenograft analysis. HCT116 cells stably containing shRNA\#2 $\left(5.0 \times 10^{6}\right.$ cells resuspended in $200-\mu 1$ serum-free RPMI) were subcutaneously injected into the flanks of 7-week-old female $n u / n u$ mice. When tumor volumes reached $150-200 \mathrm{~mm}^{3}$, the animals were provided water with or without $2 \%$ doxycycline ("Dox") ( $n=5$ mice per group). At 28 days, the relative levels of MAGE-A12 mRNA were measured by qRT-PCR analysis (A). A representative image of xenograft tumors (B). Tumor volumes $\left(\mathrm{mm}^{3}\right)$ were measured every other day $(\mathrm{mean} \pm \mathrm{SD} ; \mathrm{n}=5, \mathrm{C}) . * * * \mathrm{p}$ $<0.001$ by t-test. (D) Tumors recovered from sacrificed mice at day 19 were weighed (mean = horizontal line). Differences between Doxtreated $(+)$ versus Dox-untreated (-) groups were statistically significant $(* \mathrm{p}<0.05$, by t-test). 

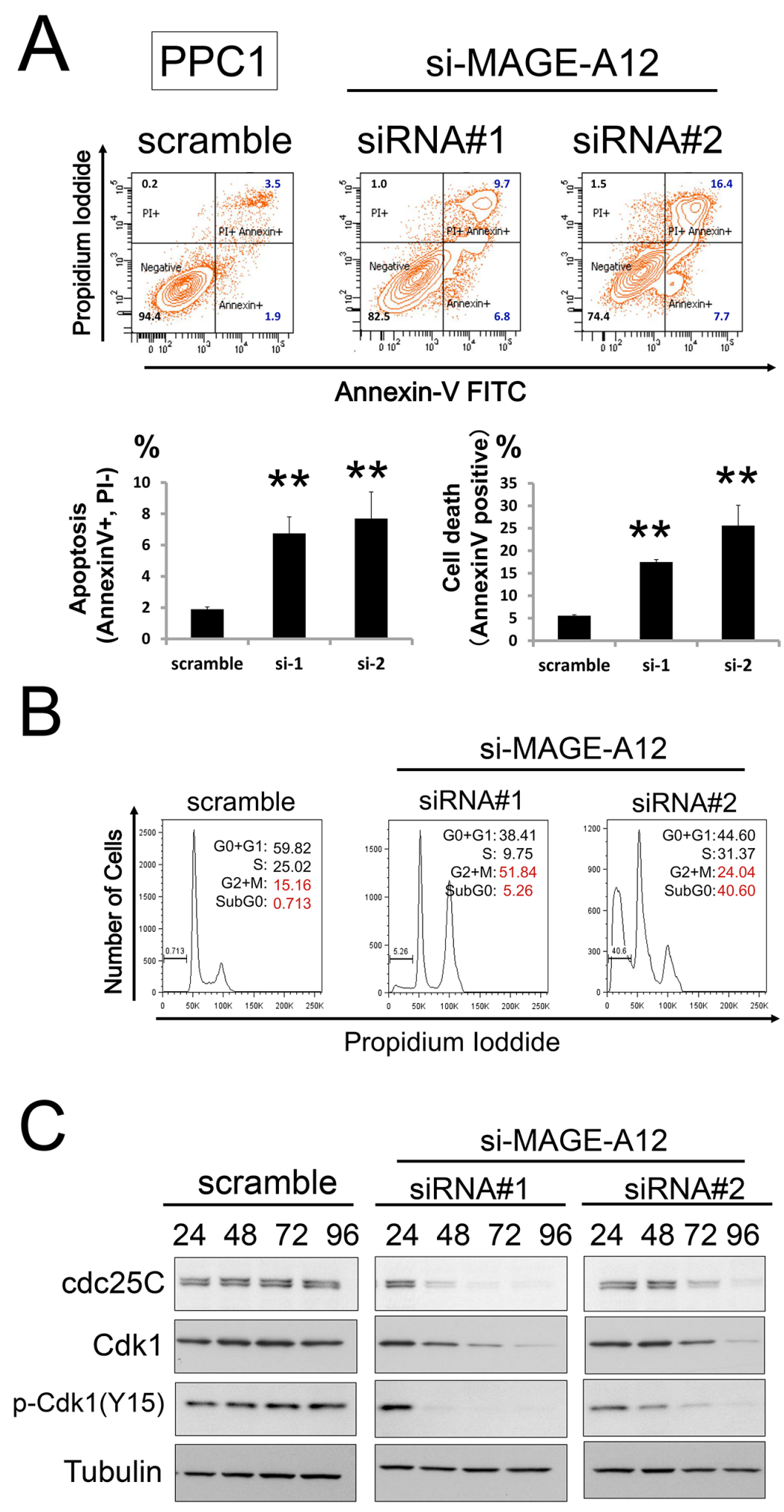

Figure 3: MAGEA12 regulates the cell cycle and apoptosis of cancer cells. (A, B) Prostate cancer PPC1 cells were transfected with scrambled RNA or two different siRNAs targeting MAGE-A12 (siRNAs\#1, \#2). At 48 hours, FACS analysis was performed to assess the percentage of cells binding Annexin V. The \% Annexin V-positive cells (A: apoptosis, B: cell death) were then assessed (mean \pm SD; $n$ $=3, * * \mathrm{p}<0.01$ ). (C) PPC1 cells transfected with siRNAs were cultured for 48 hours, followed by FACS analysis of DNA content. Data represent relative DNA (propidium iodide fluorescence; $x$-axis) versus relative cell number (y-axis). (D) PPC1 cells transfected with control or siRNAs targeting MAGE-A12 were incubated for the indicated hours. Cell lysates were harvested, followed by immunoblotting with the indicated antibodies. 

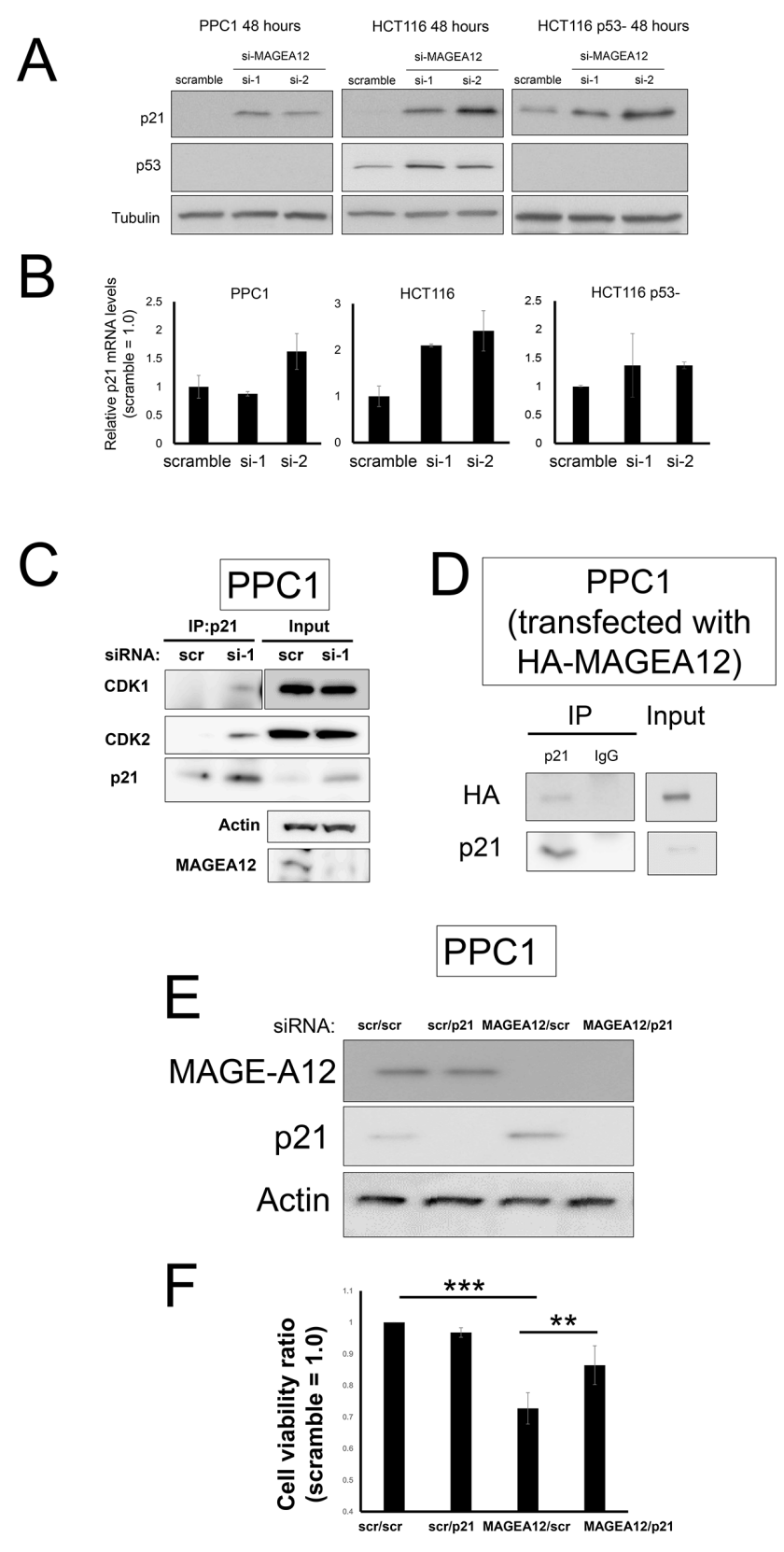

Figure 4: MAGE-A12 knockdown leads to accumulation of p21. (A, B) PPC1, HCT116, and isogenic p53-deficient HCT116 (HCT116 p53-) cells were transfected with control RNA or two different siRNAs targeting MAGE-A12. At 48 hours, cell lysates were prepared, normalized for total protein content, and analyzed by immunoblotting using antibodies for p21 (top), p53 (middle), and tubulin (bottom) (A). Also, mRNAs were extracted and levels of p21 mRNA were measured by qRT-PCR, with normalization relative to GADPH (mean $\pm \mathrm{SD} ; \mathrm{n}=2$ ) (B). (C) PPC1 cells were reverse-transfected with control or siRNA targeting MAGE-A12 (si-1). At 48 hours, the cells were lysed in buffer containing $1 \%$ NP-40, and the cell lysates were subjected to immunoprecipitation (IP) with antibodies to p21 and the resulting precipitates were subjected to immunoblot analysis with antibodies to the indicated proteins. A portion (5\%) of the lysates ("input") was also subjected directly to immunoblot analysis with the same antibodies. (D) PPC1 cells were transfected with the pcDNA3-HA-MAGE-A12 vector. At 48 hours, the cells were lysed in buffer containing 1\% NP-40, and the cell lysates were subjected to immunoprecipitation (IP) with antibodies to p21 or control rabbit IgG and the resulting precipitates were subjected to immunoblot analysis with anti-HA antibody. A portion (5\%) of the lysates ("input") was also subjected directly to immunoblot analysis. (E, F) PPC1 cells transfected with scramble-control RNA, or siRNAs targeting MAGE-A12 and p21 in various combinations as indicated (5 nM each, total siRNA concentration of $10 \mathrm{nM}$ ). At 72 hours, cell lysates were prepared, normalized for total protein content, and analyzed by immunoblotting using antibodies for MAGE-A12 (top), p21 (middle), and beta-actin (bottom) (E). (F) Cellular ATP levels were measured, with data expressed as the ratio of values for cells transfected with various siRNAs to values for the control $\operatorname{siRNAs}(\mathrm{mean} \pm \mathrm{SD}$; $\mathrm{n}=3$ ). $* * \mathrm{p}<0.01, * * * \mathrm{p}<0.001$ by t-test. 
Cdk2 (Figure 4C), suggesting that p21 retains its ability to suppress target Cdks. Interaction of MAGE-A12 with p21 was confirmed by a co-immunoprecipitation analysis using HA-tagged MAGE-A12 and endogenous p21 (Figure 4D). To confirm the functional role of p21 in proliferation and cell cycle arrest caused by MAGE-A12 knockdown, PPC1 cells were transfected with siRNAs targeting MAGE-A12, p21, or both, as well as with various control synthetic RNAs. Immunoblot analysis of the transfected cells confirmed MAGE-A12 and p21-knockdown after transfection (Figure 4E). In a cell viability assay, p21 knockdown restored cell viability in MAGE-A12 knockdown PPC1 cells (Figure 4F). Moreover, pcDNA3-vector-mediated p21 overexpression led to proliferation arrest and apoptosis in PPC1 cells (Supplementary Figure 8).

\section{MAGEA12 regulates p21 ubiquitination}

p21 levels are regulated by ubiquitination/ degradation mechanisms as well as by transcriptional regulation (Supplementary Figure 9A) [16]. The accumulation of $\mathrm{p} 21$ without the up-regulation of $\mathrm{p} 21$ mRNA strongly suggests that MAGE-A12 is involved in the process of p21 ubiquitination/degradation. MAGE-A family proteins have been reported to affect the ubiquitination of several proteins [9]. Thus, the effect of MAGE-A12 on p21 protein stability was examined with cyclohexamide $(\mathrm{CHX})$ chase experiments. PPC1 and HCT116 cells transfected with siRNA targeting MAGE-A12 or a scramble-control were cultured for 48 hours, whereupon the cells were treated with $25 \mu \mathrm{g} / \mathrm{ml}$ cyclohexamide and the rate of p21 turnover was monitored (Supplementary Figure 9B). Endogenous p21 protein levels were significantly more stable in MAGE-A12 knockdown PPC1 cells than in control PPC1 cells, demonstrating that MAGE-A12 down-regulates p21 in a post-translational manner and showing that MAGE-A12 knockdown stabilizes the p21 protein. To examine whether MAGE-A12 is associated with the ubiquitination of p21, HEK293T cells were co-transfected with HAubiquitin, Myc-p21, and FLAG-MAGE-A12, followed by immunoprecipitation using anti-Myc antibody and by SDS-PAGE/immunoblotting analysis of the specimens. The ubiquitination of p21 was elevated in MAGE-A12overexpressing cells (Supplementary Figure 9C). The ubiquitination of p21 appears to be mono-ubiquitination, which was recently reported to be associated with protein degradation [17].

\section{DISCUSSION}

The present study showed that MAGE-A12 is expressed in only certain cancer cells and that it promotes

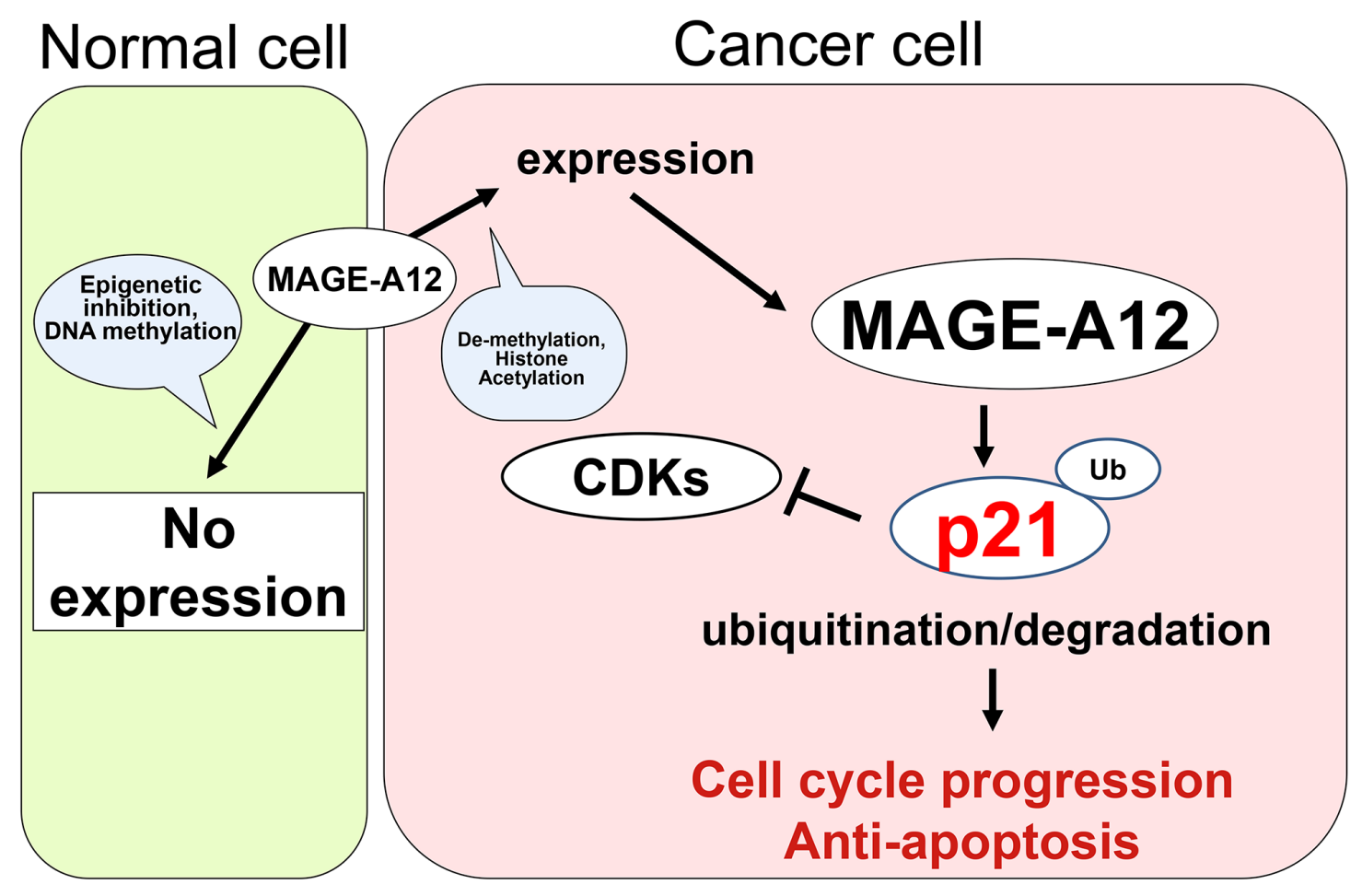

Figure 5: Model of the interaction between MAGE-A12 and p21 in cancer cells. MAGE-A12 does not express in normal cells other than in the testes because of epigenetic inhibition (DNA methylation). However, in cancer cells, MAGE-A12 is expressed due to demethylation or histone acetylation. MAGE-A12 plays a crucial role in the ubiquitination/degradation of tumor suppressor p21, which leads to cell-cycle progression and anti-apoptosis. 
the ubiquitination/degradation of tumor suppressor p21 (Figure 5). It has been known that Type I MAGE genes are located on the $\mathrm{X}$ chromosome and are expressed in the testes and placenta. The expression of Type I MAGE genes is silenced by DNA methylation; however, in malignant tumors, aberrant expression is observed. To date, the main focus on MAGE proteins has been on their potential for an anti-tumor vaccine. Concerning the physiological functions of MAGE family proteins, several studies show clues. Genome-wide yeast two-hybrid studies identified interactions between MAGE proteins and really interesting new gene (RING) proteins [18]. In 2010, MAGE proteins reported to interact with RING proteins and enhance E3 ligase mediated ubiquitination [9]. Moreover, in breast cancers, MAGE-A2, which is up-regulated in tamoxifen-resistant tumors, localizes to the nucleus and forms complexes with p53 and estrogen receptor alpha, resulting in repression of the p53 pathway [11]. Recently, MAGE-C2 has been reported to bind Rbx1 and regulate the cyclin E expression [19]. However, there are no detailed reports on the functions of MAGE-A12. In this report, we identified p21 as a target protein for the MAGE-A12-related ubiquitination pathway and showed that $\mathrm{p} 21$ levels are regulated by MAGE-A12 posttranscriptionally.

Our results also indicated that the accumulation of endogenous p21 induced by MAGE-A12 knockdown leads to G2/M arrest in tumor cells. Recently, Kreis et al. reviewed the role of p21 in the cell cycle and cell division in detail [20]. p21 is required in order to maintain G2 arrest by blocking the interaction of Cdk1 with Cdk activating kinase [21], which suggests that p21 accumulation is sufficient to arrest the cell cycle. That study is consistent with the present results, in which p21 that accumulates as a result of MAGE-A12 knockdown binds to Cdk1/ $\mathrm{Cdk} 2$. Although the function of p21 during the G2/M phases has not been fully elucidated, our study suggests that MAGE-A12 is associated with p21 degradation at the $\mathrm{G} 2 / \mathrm{M}$ phases.

Clinically, much effort has been directed towards MAGE-targeted immune therapies, but without success. In clinical trials for a lung cancer treatment, recombinant MAGE-A3 protein did not afford any greater disease-free survival than a placebo [22]. What is more, unanticipated death has been reported in T-cell-based anti-MAGE therapies [23, 24]. These large clinical studies revealed that immunotherapies using MAGE family proteins have some risks; thus, other approaches targeting MAGE family members directly should be developed. The aberrant expression of MAGE family proteins is common in various cancers; thus, MAGEA-targeted therapeutics will be applied to a wide range of cancer types. However, to date, no drugs have been reported to inhibit the interaction between MAGE-A family proteins and E3 ligases. Our study and previous studies suggest that
MAGE-A proteins function as scaffold proteins for the degradation machinery of tumor suppressors (p53, p21). Therefore, the drug discovery of inhibitors targeting the interactions between MAGE proteins and their binding proteins could be a novel strategy for cancer therapies. To achieve such therapeutics, we need more investigations on the mechanisms of MAGE family proteins, including the protein binding sites and protein modifications. Furthermore, in vivo siRNA therapies targeting cancer cells have recently been developed [25]. Since the oncogenic functions of MAGE family proteins have been unmasked, such genes could be potential targets for siRNA therapies.

\section{METHODS}

\section{Reagents and antibodies}

The Cell Titer Glo cell viability assay kit was purchased from Promega. RNAiMAX and Lipofectoamine 2000 were obtained from Life Technologies. Opti-MEM was purchased from GIBCO. RPMI and DMEM media were purchased from Cellgro. Pre-designed small interfering RNA (siRNA) directed against human MAGE-A12 (silencer select, siRNA\#1 (s8451): 5'-CACUCUAUUCUGUAAAUUU-3', siRNA\#2 (s8453): 5'-GGAGACGAGCUUCCAAGUA-3'), CDKN1A (p21, siRNA\#1: s417), and a negative scramble control (\#1) were purchased from Life Technologies. MG132 was purchased from Calbiochem. Antibodies against MAGE-A12 (mouse monoclonal, ab87973, Abcam), p53 (Ab-6, Merck), Cdk1 (610037, BD), Cdk2 (610145, BD), phospho-cdc2 (Y15) (\#9111, CST), cdc25C (\#4688, CST), alpha-tubulin (T5168, Sigma), FLAG (M2, Sigma), HA (3F10, Roche), Myc (9E10, Roche), betaactin (Sigma), horseradish-peroxidase (HRP)-conjugated secondary antibodies (GE Health Care), and Alexa Fluor 488/594-conjugated secondary antibodies (Life Technologies) were purchased from the indicated sources.

\section{Cell lines and cell culture}

PPC1, HCT116, and HCT116 p53-/- were cultured in RPMI or DMEM supplemented with $10 \%$ FBS (Sigma) and maintained at $37^{\circ} \mathrm{C}$ in a humidified atmosphere of $5 \%$ $\mathrm{CO}_{2}$ and $95 \%$ air.

\section{Plasmids}

Full-length cDNAs encoding human MAGE-A12, CDKN1A (p21), and ubiquitin were purchased from Open Biosystems. Expression plasmids for various proteins were constructed in the pcDNA3 vector for transfection. Proper plasmid construction was confirmed by restriction enzyme digestion and DNA sequencing. DNA transfection was performed using Lipifectoamine 2000 according to the manufacturer's instruction (Life Technologies). 


\section{Cell viability assays using ATP measurement}

Cell Titer Glo (Promega) was used for cell viability estimation. Cells were plated at a density of 5,000 10,000 cells per well in $100 \mu \mathrm{L}$ and cultured for 48 or 72 hours with or without treatment. The plates were then removed from the incubator and allowed to equilibrate to room temperature for about 10 minutes. Cell Titer Glo solution was added at $100 \mu \mathrm{l}$ per well, and the plates were kept in the dark for 15 minutes before the luminescence was read by luminometer (Luminoskan Ascent; Thermo Scientific Corporation).

\section{Analysis of apoptosis}

Cells were processed using the Annexin V-PI apoptosis assay kit (FITC-annexin V/ propidium iodide staining) according to the manufacturer's protocol (Life Technologies) and 20,000 events were analyzed by flow cytometry using FACS Aria (Becton Dickinson).

\section{RNA interference}

For transient knockdown, cells were transfected with siRNA duplexes by a reverse transfection method using Lipofectoamine RNAiMAX according to the manufacturer's instructions (Life Technologies). For each gene, up to 2 different siRNAs sequences were used. To confirm the siRNA silencing of genes, total protein or RNA was isolated 48 or 72 hours after transfection and was analyzed by immunoblotting or quantitative RT-PCR, respectively. The detailed protocol is provided below.

\section{Tet-inducible short hairpin RNA constructs, lentivirus and infection}

A

(CAACAAGATGAAGAGCACCAA), MAGE-A
shRNA\#1 $\quad$ (GAGTGTGTTGGAGGCATCTGA)
and shRNA
(AGACGAGCTTCCAAGTAGCAC) were cloned
inducible pLKO-Tet-On puromycin vectors as previous
described [26, 27]. Lentiviral supernatants were genera
according to an established protocol. Cells were selecter
by $1 \mu \mathrm{g} / \mathrm{ml}$ puromycin (MP Biomedicals) and expand
shRNA was induced by the addition of $100 \mathrm{ng}$
doxycycline (Sigma) to the medium.
Extraction of total RNA and quantitative RT-
PCR analysis

We isolated total RNA from cultured cells and xenograft tumors using the RNeasy Plus Mini Kit (Qiagen). RNA concentrations were measured spectrophotometrically and samples were stored at $-80{ }^{\circ} \mathrm{C}$ until being used for RTPCR. We reverse-transcribed RNA by using Superscript III (Life Technologies) according to the manufacturer's instructions. Complementary DNA samples were analyzed by the SYBR green system (Promega). The sequences for primers specific for human MAGE-A2, MAGE-A3, MAGE-A6, MAGE-A12, human CDKN1A (p21), and the control housekeeping genes for human GAPDH are as follows:

Human GAPDH

Forward: 5'- GAAGGTGAAGGTCGGAGTC -3'

Reverse: 5'- ATGGGATTTCCATTGATGAC -3'

Human MAGE-A2

Forward: 5'- GGTCGACAGATGCAGTGGT -3'

Reverse: 5'- CTGTCCCCCTCAGAACCTC -3'

Human MAGE-A3

Forward: 5'- GTGAGGAGGCAAGGTTCTGA -3'

Reverse: 5'- GGGCAATGGAGACCCACT -3'

Human MAGE-A6

Forward: 5'- GCCCTCTCACTTCCTCCTTC $-3^{\prime}$

Reverse: 5'- GAGCTGGGCAATGGAGAC -3'

Human MAGE-A12

Forward: 5'- GTGGTCCTAAGATCTACCAAGCA -3'

Reverse: 5'- AGGGCAGCAGGTAGGAGTG -3'

Human CDKN1A (p21)

Forward: 5'- CCGAAGTCAGTTCCTTGTGG -3'

Reverse: 5'- CATGGGTTCTGACGGACAT -3'

All experiments were performed in either duplicate or triplicate and were normalized with respect to GAPDH levels.

\section{Laemmli SDS-PAGE, immunoblotting and immunoprecipitation}

Cells were washed twice with PBS and harvested with radioimmunoprecipitation assay (RIPA) buffer composed of $20 \mathrm{mM}$ Tris- $\mathrm{HCl}, \mathrm{pH} 7.5,150 \mathrm{mM} \mathrm{NaCl}, 0.1 \mathrm{mM}$ EDTA, $1 \%$ Nonidet P-40, 0.1\% SDS, $5 \mathrm{mM} \mathrm{NaF}$ and an EDTAfree complete protease cocktail tablet (Roche). The cells were left on ice for $20 \mathrm{~min}$ and centrifuged at 14,000 x $\mathrm{g}$ for 10 minutes. Protein concentrations were measured using the Bio-Rad protein assay kit (Bio-Rad). For Laemmli SDS-PAGE, proteins were separated on SDS-PAGE 4-15\% gradient gels (Life Technologies) and transferred onto nitrocellulose membranes (Bio-Rad). Proteins were transferred to nitrocellulose membranes, which were blocked for 1 hour in Tris-buffered saline (TBS) with $0.05 \%$ Tween- 20 and $5 \%$ non-fat dry milk, and were then incubated overnight at $4{ }^{\circ} \mathrm{C}$ with primary antibodies diluted in blocking buffer. Membranes were rinsed three times in TBS with $0.05 \%$ Tween- 20 and incubated with secondary HRP-conjugated antibodies for 1 hour at room temperature. An enhanced chemiluminescence (ECL) method (GE Health Care) was used for detection.

For immunoprecipitation (IP), cells were lysed in $1 \%$ NP-40 lysis buffer ( $20 \mathrm{mM}$ Tris-HCl, $\mathrm{pH} 7.5,150 \mathrm{mM}$ $\mathrm{NaCl}, 0.1 \mathrm{mM}$ EDTA, $1 \%$ Nonidet P-40, $5 \mathrm{mM} \mathrm{NaF}$ and an EDTA-free cOmplete protease cocktail tablet). For analysis of ubiquitination of $\mathrm{p} 21$, cells were treated with $10 \mu \mathrm{M}$ MG132 for 4 hours and lysed in RIPA buffer with 
$10 \mu \mathrm{M}$ MG132. Three milligrams of protein lysate was used for immunoprecipitation by incubation with $2 \mu \mathrm{g}$ of the antibody for 2 hours at $4{ }^{\circ} \mathrm{C}$. Then, $30 \mu \mathrm{l}$ of protein $\mathrm{G}$ resin (Life Technologies) was added for 1 hour at $4^{\circ} \mathrm{C}$ and the IPs were washed four times with lysis buffer. Sample buffer was then added, and the beads were boiled for 10 minutes at $100{ }^{\circ} \mathrm{C}$. Samples were then analyzed by SDSPAGE followed by immunoblotting.

\section{Clonogenic assay}

Cells with Tet-inducible shRNA targeting MAGE-A12 were seeded at 500 cells per well in 6-well $(35 \mathrm{~mm})$ dishes. The cells were cultured with $(\mathrm{ON})$ or without $(\mathrm{OFF})$ doxycycline $(100 \mathrm{ng} / \mathrm{ml})$ for 14 days before being fixed and stained. For fixation, the cells were washed twice with PBS (pH 7.4) and were incubated with methanol at $-20^{\circ} \mathrm{C}$ for 20 minutes. Then, the cells were washed with PBS and incubated with $0.5 \%$ crystal violet dye in $25 \%$ methanol for 15 minutes. Finally, the dishes were immersed in tap water to remove excess crystal violet. A colony was defined as consisting of at least 50 cells.

\section{Cell growth assay}

To measure cell growth rates, $1.0 \times 10^{5}$ cells with Tetinducible shRNA targeting MAGE-A12 were plated onto 60 -mm-diameter plates with complete media with $10 \%$ FBS. After 24 hours, the culture media was changed to that with $(\mathrm{ON})$ or without (OFF) doxycycline $(100 \mathrm{ng} / \mathrm{ml})$. The numbers of cells were counted at $1,3,5$, and/or 7 days after seeding using the Countess automated cell counter (Life Technologies). In siRNA experiments, $1.0 \times 10^{5}$ cells were reverse-transfected with siRNAs targeting MAGE-A12 or the scramble control. At 72 hours, the number of cells was counted.

\section{Cell cycle analysis by FACS}

Cells were trypsinized, washed twice with PBS, fixed with cold $70 \%$ ethanol, and suspended in $100 \mu$ of PBS with propidium iodide $(20 \mu \mathrm{g} / \mathrm{ml})$ and ribonuclease A $(10 \mu \mathrm{g} / \mathrm{ml})$. Then they were subjected to cell cycle analysis using FACS Aria (Becton Dickinson) and ModFitLT Software. A total 10,000 events were analyzed.

\section{Tumor xenograft experiments}

All animal experiments were approved by the Institutional Animal Care and Use Committee of the Sanford-Burnham-Prebys Medical Discovery Institute. HCT116 cells $\left(5.0 \times 10^{6}\right)$ with Tet-inducible shRNA targeting MAGE-A12 (shRNA\#2) resuspended in 200- $\mu$ l serum-free DMEM were injected subcutaneously into the flanks of 7-week-old female $n u / n u$ mice using a 25-gauge needle. When the tumor volumes reached $150-200 \mathrm{~mm}^{3}$, the animals were given water with $2 \%$ doxycycline.
Tumor size was measured every three days using calipers, and the tumor volume was calculated using the following formula: (long axis $\mathrm{x}$ short $\mathrm{axis}^{2}$ )/2. The endpoint was set as tumor growth exceeding $1000 \mathrm{~mm}^{3}$ (Day 27 in the HCT116 xenograft).

\section{Statistical analysis}

Statistical analysis was performed using the Excel add-in software Statcel® (OMS Ltd., Tokyo, Japan). Means and standard deviation (SD) were calculated statistically from three determinations. The data are expressed as mean $\pm \mathrm{SD}$. The chi-square goodness-of-fit test was used to determine whether the data were normally distributed. In this study, all the data were normally distributed; thus, we used t-tests (Student's or Welch's t) to assess the statistical significance of differences between various samples. $\mathrm{p}<0.05$ was considered significant.

\section{Gene expression}

Individual cancer data sets were downloaded from Oncomine (https:/www.oncomine.org), as previously described $[26,28]$. The microarray dataset of MAGE-A12 mRNA expression in normal human organs was accessed using the Oncomine database [29] and was downloaded from NEBI-GEO (series GSE7307, measured by Human Genome U133 Plus 2.0 Array). Regarding box plots, the heavy line within the box indicates the median expression value for each organ. The upper and lower edges of the box show the $75^{\text {th }}$ and $25^{\text {th }}$ percentiles of the distribution, and the ends of the whiskers from the box indicate the $90^{\text {th }}$ and $10^{\text {th }}$ percentiles, respectively. The black dots represent maximum and minimum values. A student's t-test gave a P-value for the comparison of MAGE-A12 expression between testis and the others.

Publicly available datasets were identified using webbased NextBio Software (http://www.nextbio.com, [30]). The bar graph was derived from a previous study (study ID: TCGA - aggregate samples of 14 cancer RNA-seq expression profiles). The MAGE-A12 expression data were measured by Illumina Genome Analyzer RNA Sequencing Version 2 analysis and were obtained from patients in The Cancer Genome Atlas (TCGA). The datasets included samples for bladder cancer tumors $(n=223)$, breast cancer tumors $(n=1018)$, head and neck cancer tumors $(n=427)$, chromophobe kidney tumors $(\mathrm{n}=66)$, kidney renal clear cell carcinomas $(\mathrm{n}=519)$, kidney renal papillary cell carcinomas $(n=172)$, liver hepatocellular carcinomas $(\mathrm{n}=482)$, prostate adenocarcinomas $(\mathrm{n}=259)$, stomach adenocarcinomas $(\mathrm{n}=285)$, and thyroid carcinomas $(\mathrm{n}=500)$.

The prognostic significance of $M A G E-A 12$ in the overall survival of lung or gastric cancers was assessed using the Kaplan-Meier Plotter (http://kmplot.com/analysis/), which employs gene expression microarray data and 
survival information of patients with lung or gastric cancers downloaded from GEO (Affimetrix HGU133A, HGU 133+2, and HGU 133A 2.0) according to our previous report [26, 31].

\section{ACKNOWLEDGMENTS}

We thank John Reed, Yoav Altman, Yasuko Matsuzawa, Ellen Toyonaga, and Hiroko Yanagi for their advice and technical assistance. Teruki Yanagi is supported by Japan Society of Promotion of Science (JSPS) research fellowships for young scientists, JSPS fellowships for research abroad, the Kanae Foundation for the Promotion of Medical Science, and the Sumitomo Life Social Welfare Service Foundation.

\section{CONFLICTS OF INTEREST}

The authors declare no conflicts of interest.

\section{REFERENCES}

1. Weon JL, Potts PR. The MAGE protein family and cancer. Curr Opin Cell Biol. 2015; 37:1-8.

2. Sang M, Lian Y, Zhou X, Shan B. MAGE-A family: attractive targets for cancer immunotherapy. Vaccine. 2011; 29:8496-8500.

3. Otte M, Zafrakas M, Riethdorf L, Pichlmeier U, Löning T, Jänicke F, Pantel K. MAGE-A gene expression pattern in primary breast cancer. Cancer Res. 2001; 61:6682-6687.

4. Grigoriadis A, Caballero OL, Hoek KS, da Silva L, Chen YT, Shin SJ, Jungbluth AA, Miller LD, Clouston D, Cebon J, Old LJ, Lakhani SR, Simpson AJ, et al. CT-X antigen expression in human breast cancer. Proc Natl Acad Sci U S A. 2009; 106:13493-13498.

5. Suyama T, Shiraishi T, Zeng Y, Yu W, Parekh N, Vessella RL, Luo J, Getzenberg RH, Kulkarni P. Expression of cancer/testis antigens in prostate cancer is associated with disease progression. Prostate. 2010; 70:1778-1787.

6. Hartmann S, Meyer TJ, Brands RC, Haubitz IR, Linz C, Seher A, Kübler AC, Müller-Richter UD. MAGE-A expression clusters and antineoplastic treatment in head and neck cancer. Int J Mol Med. 2015; 35:1675-1682.

7. Zhai X, Xu L, Zhang S, Zhu H, Mao G, Huang J. High expression levels of MAGE-A9 are correlated with unfavorable survival in lung adenocarcinoma. Oncotarget. 2016; 7:4871-4881. doi: 10.18632/oncotarget.6741.

8. Yamada R, Takahashi A, Torigoe T, Morita R, Tamura Y, Tsukahara T, Kanaseki T, Kubo T, Watarai K, Kondo T, Hirohashi Y, Sato N. Preferential expression of cancer/testis genes in cancer stem-like cells: proposal of a novel subcategory, cancer/testis/stem gene. Tissue Antigens. 2013; 81:428-434.

9. Doyle JM, Gao J, Wang J, Yang M, Potts PR. MAGERING protein complexes comprise a family of E3 ubiquitin ligases. Mol Cell. 2010; 39:963-974.
10. Yang B, O'Herrin SM, Wu J, Reagan-Shaw S, Ma Y, Bhat KM, Gravekamp C, Setaluri V, Peters N, Hoffmann FM, Peng H, Ivanov AV, Simpson AJ, Longley BJ. MAGE-A, mMage-b, and MAGE-C proteins form complexes with KAP1 and suppress p53-dependent apoptosis in MAGEpositive cell lines. Cancer Res. 2007; 67:9954-9962.

11. Wong PP, Yeoh CC, Ahmad AS, Chelala C, Gillett C, Speirs $\mathrm{V}$, Jones JL, Hurst HC. Identification of MAGEA antigens as causal players in the development of tamoxifen-resistant breast cancer. Oncogene. 2014; 33:4579-4588.

12. Mollaoglu N, Vairaktaris E, Nkenke E, Neukam FW, Ries J. Expression of MAGE-A12 in oral squamous cell carcinoma. Dis Markers. 2008; 24:27-32.

13. Wu Y, Li Y, Lin Y. Expression of MAGE-12 in lung cancer. Lung Cancer. 2002; 35:95.

14. Abbas T, Dutta A. p21 in cancer: intricate networks and multiple activities. Nat Rev Cancer. 2009; 9:400-414.

15. Gire V, Dulic V. Senescence from G2 arrest, revisited. Cell Cycle. 2015; 14:297-304.

16. Kim Y, Starostina NG, Kipreos ET. The CRL4Cdt2 ubiquitin ligase targets the degradation of p21Cip1 to control replication licensing. Genes Dev. 2008; 22:2507-2519.

17. Braten O, Livneh I, Ziv T, Admon A, Kehat I, Caspi LH, Gonen H, Bercovich B, Godzik A, Jahandideh S, Jaroszewski L, Sommer T, Kwon YT, et al. Numerous proteins with unique characteristics are degraded by the $26 \mathrm{~S}$ proteasome following monoubiquitination. Proc Natl Acad Sci U S A. 2016; 113:E4639-4647.

18. Rual JF, Venkatesan K, Hao T, Hirozane-Kishikawa T, Dricot A, Li N, Berriz GF, Gibbons FD, Dreze M, AyiviGuedehoussou N, Klitgord N, Simon C, Boxem M, et al. Towards a proteome-scale map of the human proteinprotein interaction network. Nature. 2005; 437:1173-1178.

19. Hao J, Song X, Wang J, Guo C, Li Y, Li B, Zhang Y, Yin Y. Cancer-testis antigen MAGE-C2 binds Rbx1 and inhibits ubiquitin ligase-mediated turnover of cyclin E. Oncotarget. 2015; 6:42028-42039. doi: 10.18632/oncotarget.5973.

20. Kreis NN, Louwen F, Yuan J. Less understood issues: p21(Cip1) in mitosis and its therapeutic potential. Oncogene. 2015; 34:1758-1767.

21. Smits VA, Klompmaker R, Vallenius T, Rijksen G, Mäkela TP, Medema RH. p21 inhibits Thr161 phosphorylation of Cdc2 to enforce the G2 DNA damage checkpoint. J Biol Chem. 2000; 275:30638-30643.

22. Ruiz R, Hunis B, Raez LE. Immunotherapeutic agents in non-small-cell lung cancer finally coming to the front lines. Curr Oncol Rep. 2014; 16:400.

23. Linette GP, Stadtmauer EA, Maus MV, Rapoport AP, Levine BL, Emery L, Litzky L, Bagg A, Carreno BM, Cimino PJ, Binder-Scholl GK, Smethurst DP, Gerry AB, et al. Cardiovascular toxicity and titin cross-reactivity of affinityenhanced T cells in myeloma and melanoma. Blood. 2013; 122:863-871. 
24. Morgan RA, Chinnasamy N, Abata-Daga D, Gros A, Robbins PF, Zheng Z, Dudley ME, Feldman SA, Yang JC, Sherry RM, Phan GQ, Hughes MS, Kammula US, et al. Cancer regression and neurological toxicity following anti-MAGE-A3 TCR gene therapy. J Immunother. 2013; 36:133-151.

25. Yanagi T, Tachikawa K, Wilkie-Grantham R, Hishiki A, Nagai Ko, Toyonaga E, Chivukula P, Matsuzawa SI. Lipid nanoparticle-mediated siRNA transfer against PCTAIRE1/ PCTK1/Cdk16 inhibits in vivo cancer growth. Mol Ther Nucleic Acids. 2016; 5:e327.

26. Yanagi T, Krajewska M, Matsuzawa SI, Reed JC. PCTAIRE1 phosphorylates p27 and regulates mitosis in cancer cells. Cancer Res. 2014; 74:5795-5807.

27. Wiederschain D, Wee S, Chen L, Loo A, Yang G, Huang A, Chen Y, Caponigro G, Yao YM, Lengauer C, Sellers WR, Benson JD. Single-vector inducible lentiviral RNAi system for oncology target validation. Cell Cycle. 2009; 8:498-504.

28. Yanagi T, Reed JC, Matsuzawa S. PCTAIRE1 regulates p27 stability, apoptosis and tumor growth in malignant melanoma. Oncoscience. 2014; 1:624-633. doi: 10.18632/ oncoscience. 86 .

29. Rhodes DR, Kalyana-Sundaram S, Mahavisno V, Varambally R, Yu J, Briggs BB, Barrette TR, Anstet MJ, Kincead-Beal C, Kulkarni P, Varambally S, Ghosh D, Chinnaiyan AM. Oncomine 3.0: genes, pathways, and networks in a collection of 18,000 cancer gene expression profiles. Neoplasia. 2007; 9:166-180.

30. Kupershmidt I, Su QJ, Grewal A, Sundaresh S, Halperin I, Flynn J, Shekar M, Wang H, Park J, Cui W, Wall GD, Wisotzkey R, Alag S, et al. Ontology-based meta-analysis of global collection of high-throughput public data. PLoS One. 2010; 5:e13066.

31. Szász AM, Lánczky A, Nagy Á, Förster S, Hark K, Green JE, Boussioutas A, Busuttil R, Szabó A, Győrffy B. Cross-validation of survival associated biomarkers in gastric cancer using transcriptomic data of 1,065 patients. Oncotarget. 2016; 7:49322-49333. doi: 10.18632/ oncotarget.10337. 